\title{
Paper Autonomous Navigation Control of UAV Using Wireless Smart Meter Devices
}

\author{
Kiyoshi Ueda ${ }^{1}$ and Takumi Miyoshi ${ }^{2}$ \\ ${ }^{1}$ College of Engineering, Nihon University, Koriyama, Japan \\ ${ }^{2}$ College of Systems Engineering and Science, Shibaura Institute of Technology, Saitama, Japan
}

https://doi.org/10.26636/jtit.2019.132319

\begin{abstract}
In preparation for the upcoming home delivery services that rely on Unmanned Aerial Vehicles (UAVs), we developed a new multi-hop radio network that is laid over a smart meter network transferring electric energy information only. In this network, a UAV follows, for navigation purposes, the topology of a virtual network overlaid on the physical smart meter network. We established a service management control method which does not rely on image analysis or map information processing, i.e. processes that consume precious power resources of the UAV. Instead, navigation is based on the routing technology. The current distance between the UAV and a node of the smart meter network is measured by means of the radio transmission loss value, therefore determining the position of the UAV. A two-layer network model has been proposed. One layer consists of a network of nodes in a residential area with scattered buildings - a location that is safer to navigate - while the other is an access network of nodes in a densely populated area. Then, we proposed methods to determine the direction of movement towards the next hop node on the data-link layer and the end node on the network layer, which is the target destination. We implemented a software-based test system and verified the effectiveness of the proposed methods.
\end{abstract}

Keywords-ad-hoc network, routing, smart meter network, UAV delivery.

\section{Introduction}

Unmanned Aerial Vehicle (UAV) control technology has been attracting a lot of attention recently. Introduction of UAVs is expected to bring about a reduction in labor costs and an improvement in the efficiency of home delivery services. The number of home deliveries increases every single year, and methods guaranteeing that the deliveries will be made in a secure and rapid manner need to be developed. Road traffic jams become an ever more burdensome phenomenon, as the volume of goods distributed grows. Workforce shortages caused by a decrease in the number and the aging of truck drivers become a serious social problem as well. The unmanned home delivery technology relying on mobile equipment is expected to be able to solve such problems. Home deliveries performed without visual observation of UAVs become ever more efficient. However, navigation management systems and crash prevention tech- nologies still need to be improved. Methods used to determine the position of UAVs and to ensure identical delivery routes using enormous amounts of mapping and GPS data, as well as images sourced from UAV-mounted cameras and requiring real-time analysis, have been researched. Also, some methods used to pinpoint the position of the destination are known, but they include image analysis and map information processing, which consumes precious power resources of any UAV.

On the other hand, wireless devices known as "smart, nextgeneration electricity meters" [1] have been installed in each house in Japan. These wireless devices are installed to transfer electric billing information to utility companies, relying on a energy efficient, multi-hop radio $(920 \mathrm{MHz})$ network. Such devices may be considered to be nodes of a stable multi-hop radio network, as they are present all over the country. In Japan, they were introduced in large quantities between 2016 and 2018, and according to plan, will be installed in all houses by 2023 . We study to establish a method allowing to navigate UAVs safely and automatically based on the new multi-hop radio network between these wireless device nodes (Fig. 1).

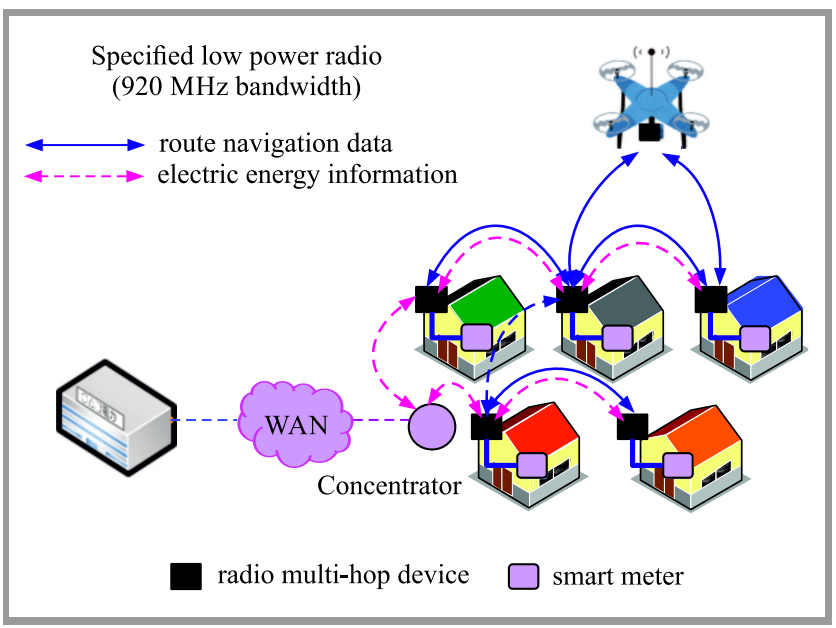

Fig. 1. Navigation based on a multi-hop radio network.

In this paper, we propose a route control protocol to build a multi-hop radio network topology in a safe, scattered res- 
idential area rather than in a densely populated area. The wireless device measures the value of radio transmission loss of the received signals. Based on the radio transmission loss value, we are able to calculate the distance to the node by applying the Friis transmission equation. The position of the UAV within the network may be determined based on the distance between the UAV and the wireless devices. We also propose a method for controlling UAVs, so that they follow a specific route. Software has been developed to enable autonomous, distributed processing by each node, without any centralized functionalities, in order to account for the scalability of the number of nodes.

Although elderly people tend not to use the Internet as often, as they find it difficult to conclude a contract with and to get the connection settings from their ISP, they usually use electricity and have smart meters installed at their homes. It is important that home delivery services are available for each house. Using the location of a smart meter is more practical than pinpointing the latitude and longitude with the use of GPS. This method is suitable not only for home delivery, but also for such services as autonomous driving.

In addition, when location-related information based on smart meters is available all over the country, even if a serious disaster, such as an earthquake occurs, and telephones and the Internet cannot be used, the said information may be expected to be used in various domains, such as searching for a smart, wireless meter of a specific house, conveying its position to the city hall, or directing the rescue services.

In Section 2 of this paper related work is presented and problems related to autonomous navigation of UAVs are shown. Three methods to solve the problems described are identified in Section 3. In Section 4, we explain, in detail, the autonomous navigation control procedure. Section 5 shows the implementation and validation results. Section 6 is devoted to a discussion and contains several suggestions.

\section{Related Works}

Amazon announced that it is planning a rapid delivery service of lightweight commercial products using UAVs [2]. Google also revealed it had been testing UAVs for two years now as part of the "Project Wing" [3] scheme, to produce drones that are capable of delivering larger items. These systems need a camera recording the surroundings all the time, which means that the protection of privacy becomes a big problem. Therefore, a method for navigating UAVs without taking photographs is required. Indoor positioning and navigating systems relying on radio systems (Wi-Fi) were studied for accuracy [4]. But these are not suitable for navigating UAVs outdoors.

The communication layer used to establish a link with numerous wireless devices has been studied as well. It relied on a radio mobile ad-hoc network (MANET) technique. The reactive type (AODV [5]) and the proactive type (OSLR [6]) have established the shortest route between the nodes of a network.
A mobile ad-hoc network is based on the two-layer model with a node cluster. In the cluster, the network layer and the upper layer are distinguished, established by the clusterby-cluster routing method [7]. Clustering is an effective method for improving the capacity to deal with node mobility, and to limit long distance communications by cascading short route messages.

\section{Proposed Method}

We propose a routing protocol to establish a safe route along a network of smart meters. The solution may be used for performing home deliveries with UAVs. We propose a control method in which the UAV communicates with the nodes, acquires information necessary for sensing the position and navigates by following the route, as if the UAV were a data packet within the network.

\subsection{Network Topology for Routing of UAVs}

The UAV route should be established with the principles of safe air travel taken into consideration. A two-layered network model has been adopted; a network of nodes in a scattered residential area (relay node), which is a safer navigation route (relay network), and an access network of nodes in a densely populated area. The overview of the network topology is presented in Fig. 2.

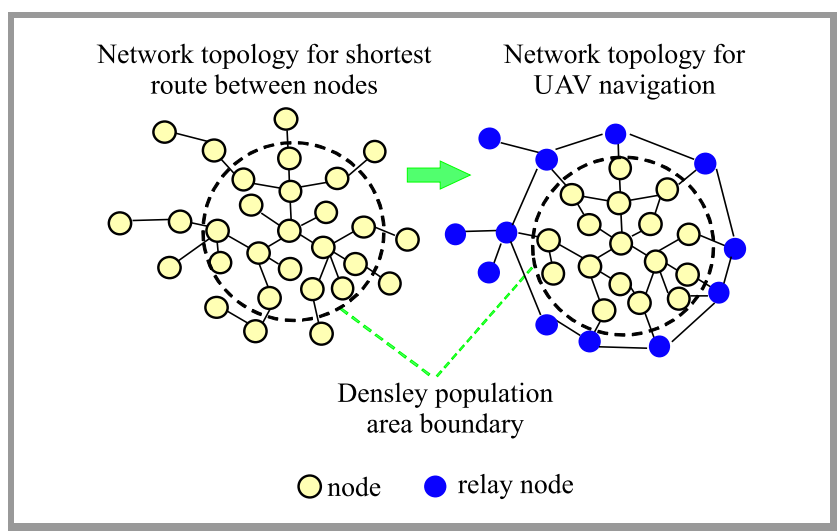

Fig. 2. UAV navigation network topology scheme.

\subsection{Routing Protocol for UAV Navigation Network Topology scheme}

Because no single delivery route is reused within a short period time for home delivery purposes, we investigated our routing protocol based on AODV - a masterpiece of the reactive type, creating a route list on demand. The route establishment process is shown in Fig. 3.

At first, information about the densely populated or the scattered residential area is set in all nodes. A parameter counting the number of passage areas is added to RREQ header of AODV. When a node transfers RREQ, the passage area counter of RREQ is incremented. 


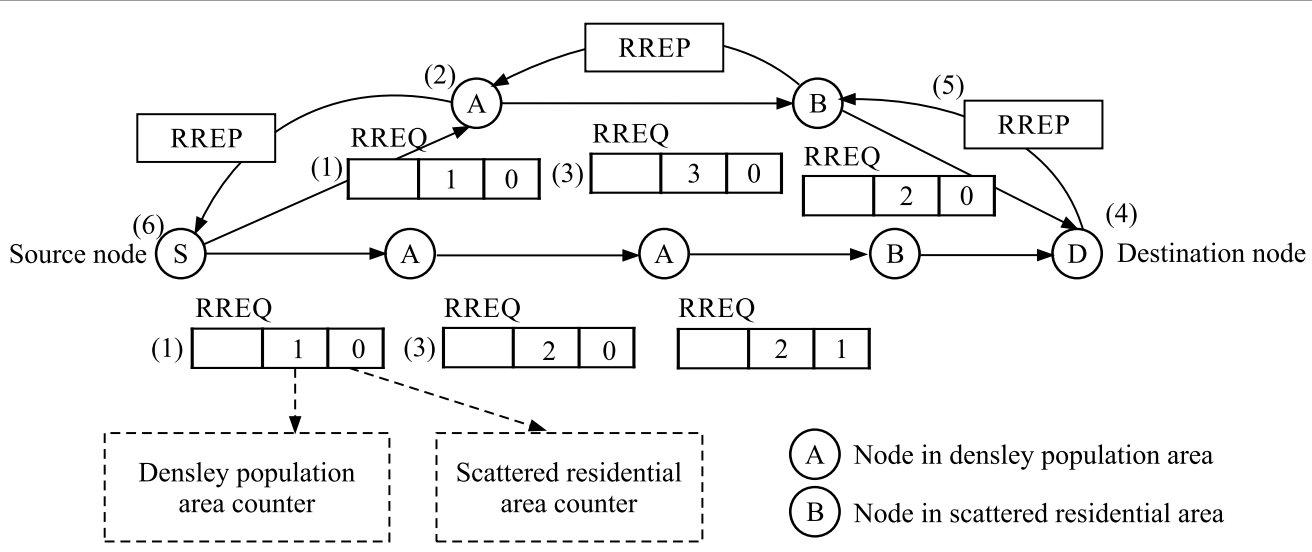

Fig. 3. Route establishment diagram.

When RREQ reaches the destination node, the number of densely populated areas and that of scattered residential areas along the route are stored. The destination node transmits RREP to the route with the smallest number of hops within densely populated areas. That is how a route avoiding densely populated areas is established. For calculating the distance between nodes, each node shall store the radio transmission loss value in the routing table, when the node receives RREQ or RREP:

1. The source node sends RREQ.

2. The node which receives RREQ adds the area count in RREQ header.

3. At step 2 above, the node which receives RREQ also records and sends the best RREQ with a lower value of densely populated area counts, compared with previous best RREQ (each node records RREQ, it received the first time, as the most suitable RREQ).

4. When RREQ signal reaches the destination node, the best route candidate is selected with the lowest number of densely populated area and residential area counts, from the numerous RREQs received.

5. The destination node repeats step 4 until time out, then it handles the elaborate route as the best root at that time. The destination node transmits RREP through that route.

6. RREP reaches the source node, and the most suitable route is established.

\subsection{Autonomous Navigation Control Method}

The proposed method maps a physical navigation route onto the virtual network model of nodes and links. UAV is connected to the multi-hop radio network and identifies the distance to each node based on the radio transmission loss value. UAV acquires information about the route to the destination node from the network, and proceeds to a position above the next hop node. When the next hop node cannot be detected due to instability of the wireless connection, UAV sends a new route request to the destination house node and another route is established. Thus, a network that is most suitable for navigation purposes is constructed based on the routing technology. Details of this approach are presented in following section.

\section{Autonomous Navigation Control Processing}

\subsection{Conditions}

The smart meter of each house is considered to be a node. Each node compiles a routing table using a routing protocol and performs home delivery based on that table. Using the routing protocol shown in Section 3.2, each node may choose the home delivery route based on the high priority of a safe, scattered residential area. The value of radio transmission loss experienced between nodes is stored in a routing table (Fig. 4 ).

The node that received RREQ (node B) sets the address of the RREQ sending node (node A) to next hop in the column where the destination is the source node (node A) in the routing table of the node itself (node B). The node (node $\mathrm{B}$ ) acquires the inter-node radio transmission loss value ( $G$ from node $A$ to node $B$ ) from the received RREQ and sets it in that column. When the node receives RREP (node A) transmitted by the destination node from the previous node (node $\mathrm{B}$ ), the node (node $\mathrm{A}$ ) sets the address of the RREP transmitting node (node B) to the next hop of the column where the destination is the destination node (node $\mathrm{C}$ ) in its own (node $\mathrm{A}$ ) routing table. The node (node $\mathrm{A}$ ) acquires inter-node radio transmission loss value (I from node $\mathrm{B}$ to node $\mathrm{A}$ ) from the received RREP and sets it in that column.

As UAV communicates with the smart meter based on the routing table, it may complete the delivery without using map information, by simply following the same delivery route that would be taken by a data packet traveling along the network. Vertical UAV movements are identified along 


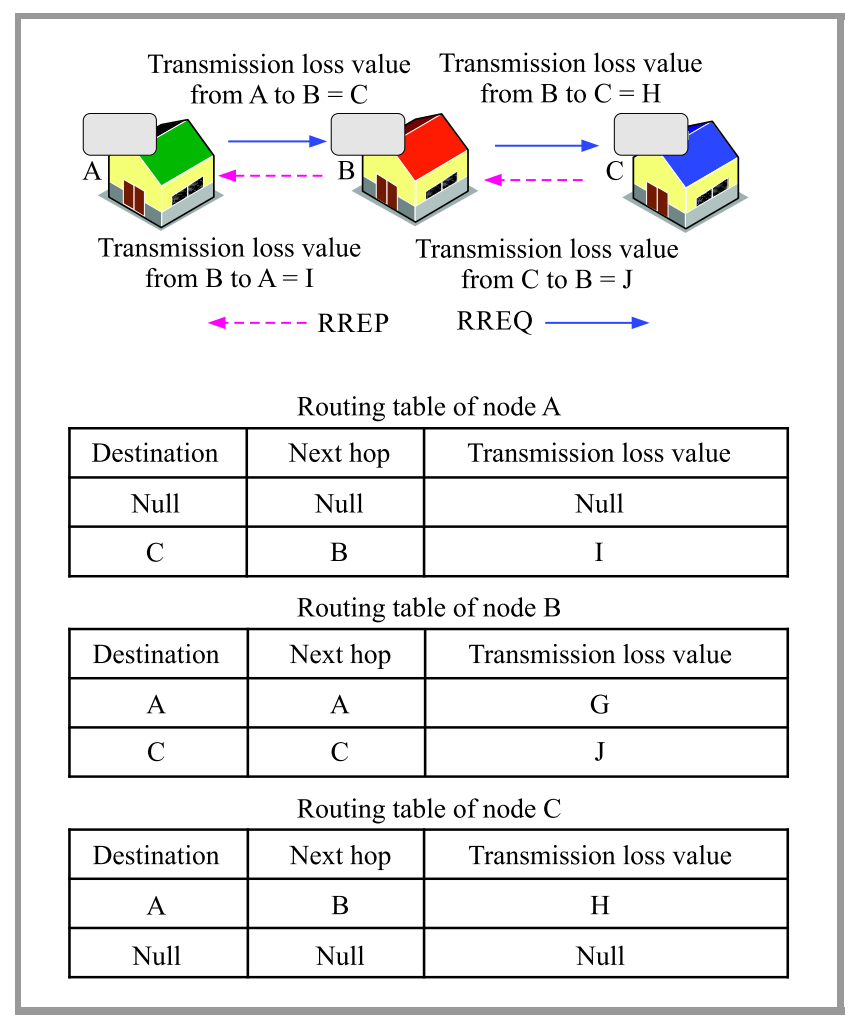

Fig. 4. Routing table.

the $\mathrm{z}$ axis, horizontal movements along the $\mathrm{y}$ axis, while back and forth movements along the $\mathrm{x}$ axis. The UAV shall be given the address of the source node and the destination node beforehand and, it is assumed that the UAV flies at a constant height $\Delta z$. We call the source node $\mathrm{S}$, the destination node $\mathrm{D}$, and the UAV $\mathrm{M}$.

\subsection{Navigation Control Flow}

UAV acquires route information from the current node. It receives information necessary for position sensing and information about navigating to the next node. UAV starts moving to the next node while sending its position repeatedly. On the way to the next node, the UAV receives position information from the current node, the next node, and the previous node to obtain the movement direction. UAV follows the route from the source node to the destination node by repeating this process.

\subsection{Proposed Signal Specifications}

Route information request. UAV sends this signal to the current node for collecting the address of the next node and the radio transmission loss value between the current node and the next node. The route information request signal stores the address of the destination node that is necessary for UAV to request information about the next node. The ID of this signal is 1 .

Route information response. The node receiving the route information request signal checks the destination node's ad- dress from the route information request signal to establish whether the specific node is the destination node or not. If the node is not the destination node, it sends a response signal to UAV, using the routing table data. The signal includes, in the data section, the address of the next node and the radio transmission loss value between the current node and the next node. The ID of this signal is 2 .

End of delivery. The node receiving the route information request signal checks the destination node's address in the route information request signal, to determine whether the node is the destination node or not. If the node is the destination node, it sends the end of delivery signal to UAV. The signal does not have a data section and the ID of this signal is 3 .

$\phi$ send. This is a signal that the UAV is transmitting to the node requiring the radio transmission loss value. The signal does not have a data section and the ID of this signal is 4 .

$\phi$ reply. This is a signal by means of which the node which is receiving the $\phi$ send signal is sending the radio transmission loss value to UAV. The signal does not have a data section and the ID of this signal is 5 .

\subsection{Route Information Request}

Data from the routing table of the current node is necessary for UAV to acquire route information. Therefore, UAV transmits the route information request signal to the current node (Fig. 5). The current node, which received the signal, transmits the route information response signal, including information about the next node, to UAV. UAV, which received the route information response signal, stores

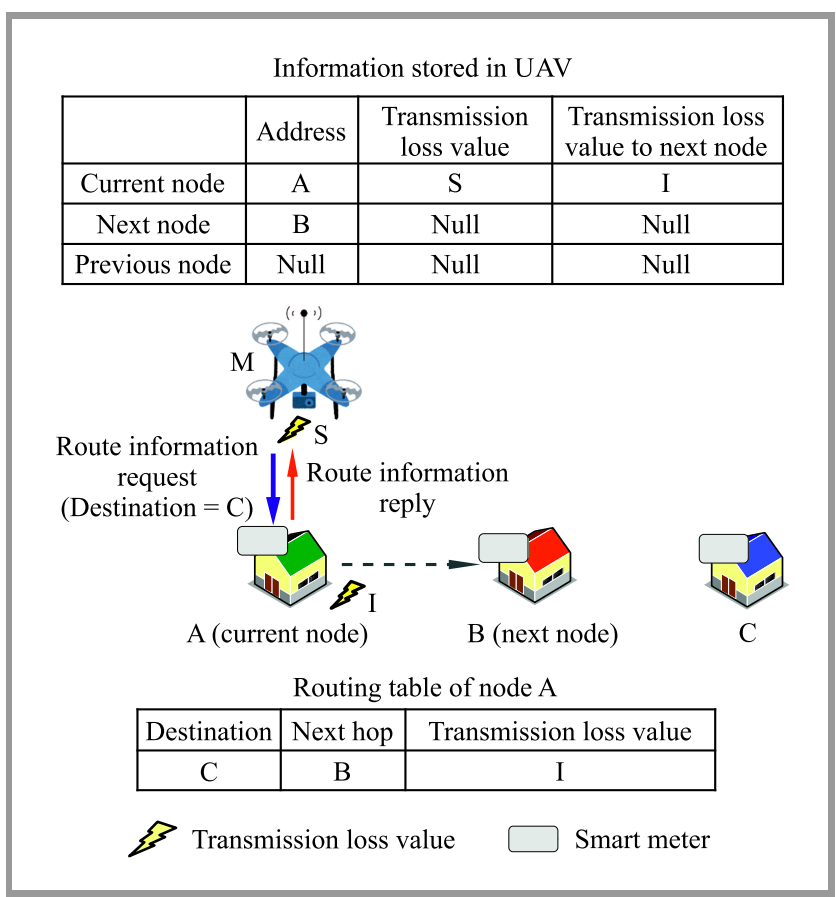

Fig. 5. Route information request. 
the radio transmission loss value to the current node, as retrieved from the packet header, as well as information in the received signal data.

\subsection{Radio Transmission Loss Value Request}

To obtain the radio transmission loss value, UAV sends the $\phi$ send signal to the current node and the next node. Then, UAV receives the $\phi$ reply signal from each node (Fig. 6). When UAV does not move, it does not send information about its position to the current node, after reception of the route information response signal, UAV obtains the value of radio transmission loss experienced while communicating with each node from the header of the $\phi$ reply signal received.

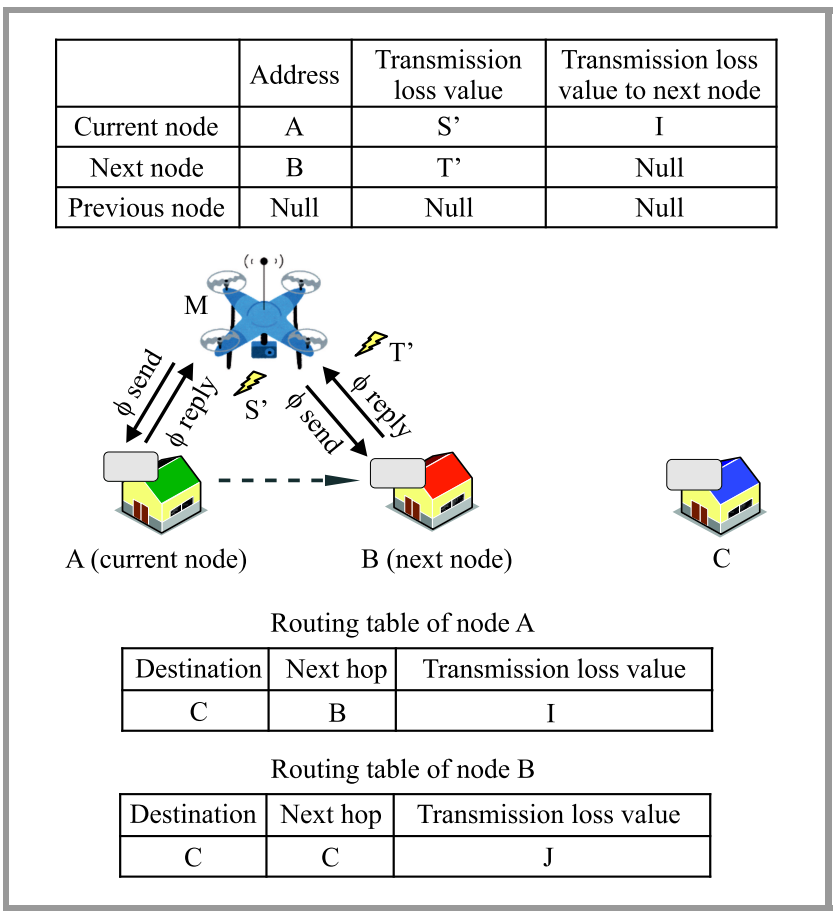

Fig. 6. Radio transmission loss value request.

\subsection{Calculating Distance from Radio Transmission Loss Value}

UAV converts the acquired radio transmission loss value into distance. The radio transmission loss value changes as the power rating becomes lower due to increasing radio transmission distance. In free space, the radio transmission loss value is calculated using the Friis transmission equation (1). At first, we determine free space transmission gain $\left(\frac{\lambda}{4 \pi d}\right)^{2}$ using the Friis transmission equation, and $\left(\frac{4 \pi d}{\lambda}\right)^{2}$, which is the reciprocal number of the free space transmission gain, is the free space transmission loss $L_{B}$ :

$$
P_{R}=P_{D} A_{R}=\left(\frac{\lambda}{4 \pi d}\right)^{2} G_{T} G_{R} P_{T},
$$

where $P_{R}$ and $P_{T}$ mean electric power, $A_{R}$ means practical antenna area, $G_{R}$ and $G_{T}$ mean antenna absolute gain,
$P_{D}$ is power density, $d$ is communications distance, $\lambda$ is wavelength.

The radio transmission $\operatorname{loss} L_{B}$ is $10 \log \frac{P_{T}}{P_{R}}$, where the terms $G_{T}$ and $G_{R}$ of the equation for obtaining $L_{B}$ are zero, assuming that an isotropic antenna (absolute gain $=1$ ) is used. Wavelength $\lambda$ is represented by $\frac{c}{f}$ using frequency $f$. Distance may be established based on radio transmission loss value by solving the expression after its conversion to distance $d(2)$ :

$$
d=\frac{4 \pi f}{c} 10^{\frac{20}{-L_{B}}}
$$

where $c$ is speed of the electric wave and $f$ is frequency. In this way, UAV may calculate the distance between nodes, as well as that between the UAV and each node.

\subsection{Establishing Position Based on Distance}

We use the Pythagorean theorem to find the length of side AM' and side BM' from $\Delta z$ of UAV height, side AM is the distance to the current node, and side BM is the distance to the next node (Fig. 7). Then, we calculate $\Delta x$, which is the distance between point $\mathrm{B}$ and the current position of the $\mathrm{UAV}$, projected onto the ground, as well as horizontal gap $\Delta y$ from the lengths of side $\mathrm{AB}$, side $\mathrm{AM}^{\prime}$ ' and side $\mathrm{BM}^{\prime}$ by using Eq. (3). In this way, we identify the position of the UAV (Fig. 8).

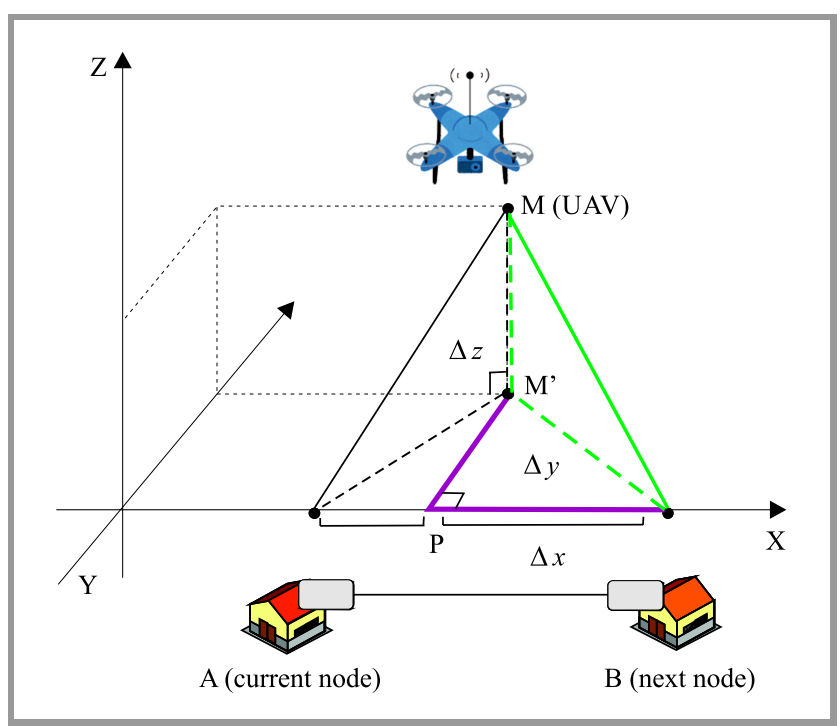

Fig. 7. Establishing the position of UAV in network 1.

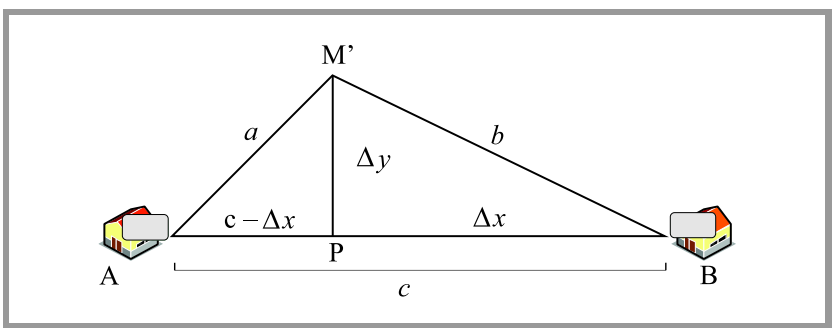

Fig. 8. Establishing the position of UAV in network 2. 


$$
\begin{gathered}
\left\{\begin{array}{c}
(c-\Delta x)^{2}+\Delta y^{2}=a^{2} \\
\Delta x^{2}+\Delta y^{2}=b^{2}
\end{array}\right. \\
\Delta x=\frac{-a^{2}+b^{2}+c^{2}}{2 c}, \\
\Delta y=\sqrt{b^{2}-\Delta x^{2}} .
\end{gathered}
$$

UAV stores the $\Delta x$ and $\Delta y$ values as old_x, old_y until the next measurement.

\subsection{Navigation Control}

After detection of the position has been accomplished, UAV moves to the next node after time unit $\Delta t$ and detects its position again. UAV adjusts the position and moves in the right direction by comparing old_ $x$ with $\Delta x$ and old_y with $\Delta y$.

Movement control along $\mathbf{y}$ axis. The tolerance threshold of $\Delta y$ (right and left movements) has been set in UAV beforehand. When the $\Delta y$ calculated is higher than the threshold value, UAV moves $\Delta \mathrm{y}$ to the right or to the left, until $\Delta y$ becomes lower than the threshold set, before sensing its next position. The first movement is always to the right, and then the value of $\Delta y$ is compared with the value of old_y. Where old_y $>\Delta y$, the value of $\Delta y$ is decreasing and UAV is moving in the right direction. Where old $y<\Delta y$, the value of $\Delta y$ is increasing. This means that UAV is moving in the opposite way, and it should change the direction.

Movement control along $\mathbf{x}$ axis. UAV adjusts its movement along $\mathrm{x}$ axis comparing the value of $\Delta x$ with the value of old_ $x$, as it did in the scenario with y axis. If old_ $x>\Delta x$, the value of $\Delta x$ is decreasing and UAV is moving to the right direction. If $o l d_{-} x<\Delta x$, that means the value of $\Delta x$ is increasing, therefore, the UAV is moving in the opposite way. Then, UAV repeats position sensing, calculates the movement and approaches the next node.

\subsection{Updating Current and Next Node}

When $\Delta x$ becomes " 0 ", UAV may arrive at the next node. Then, the current node should be updated. At first, UAV stores the current node's address as the previous node's address, sends a route information request signal to the next node, then receives the route information response signal from the next node (Fig. 9).

With this procedure, UAV considers the node to route information request signal has been sent as the new current node, and then UAV finds the new next node based on the route information response signal. After that, UAV measures the radio transmission loss value, establishes its position based on distance and moves to the next node.

\subsection{Turns}

After updating the current and the next nodes, UAV turns toward the next node. At this time, UAV may receive radio

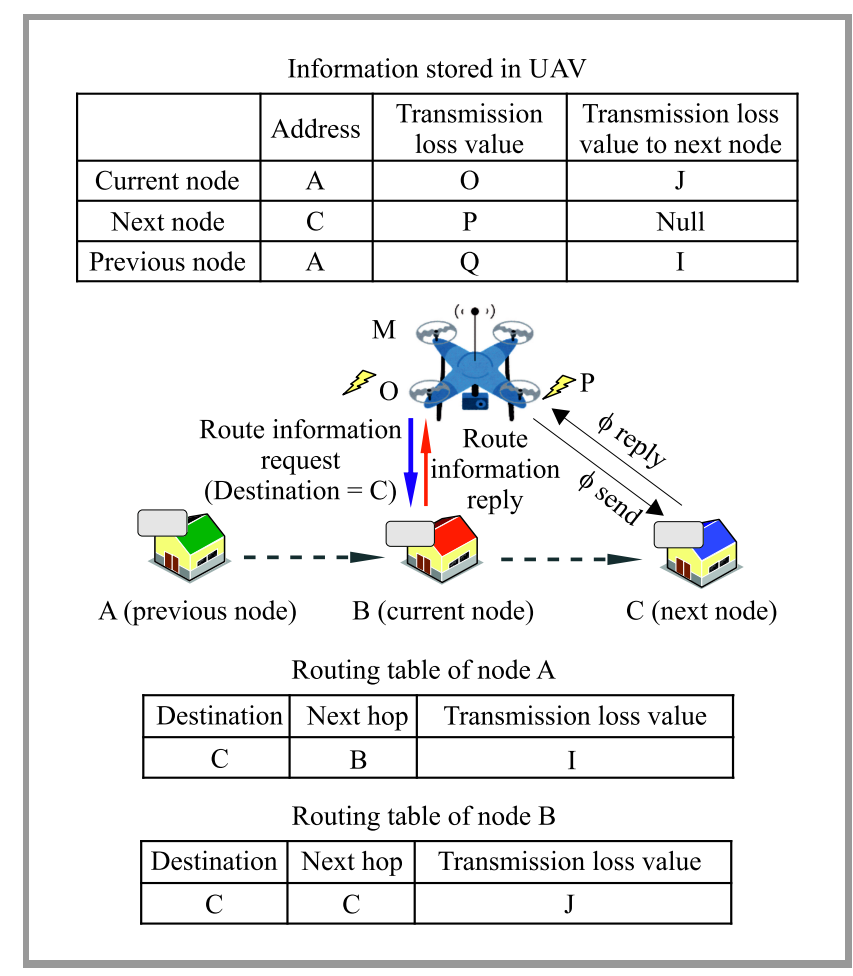

Fig. 9. Updating current and next node.

waves from the previous node, the current node and the next node. At first, UAV moves from the position above the current node to the inside of the angle between the previous and the next node. UAV moves a little to the left or the right (y axis direction), which is the direction that the radio transmission loss value decreases, and then moves the same distance backward along axis $\mathrm{x}$. At that position, UAV calculates the lengths of sides AM', BM' and CM' using the Pythagorean proposition, with height $\Delta \mathrm{z}$ and distances from UAV to the previous and the next node taken into consideration (Fig. 10).

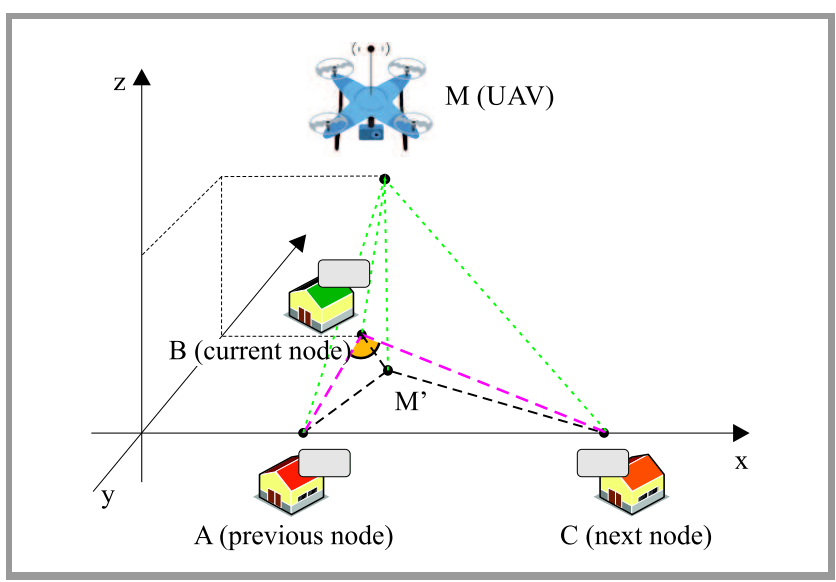

Fig. 10. Turns.

Then, UAV is capable of calculating $\angle A B M$ ' using the Law of cosines (6), based on lengths of side AB, side AM', and 


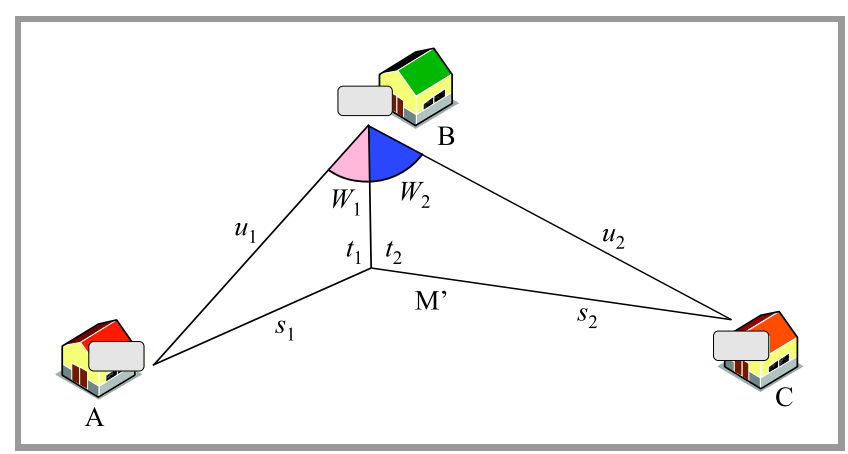

Fig. 11. Calculation of the angle by the law of cosines.

side BM' (Fig. 11). Similarly, UAV may calculate $\angle \mathrm{M}^{\prime} B C$ based on lengths of side BC, side BM', and side CM':

$$
\cos W=\frac{t^{2}+u^{2}-s^{2}}{2 t u} .
$$

The angle of rotation of UAV may be calculated by subtracting the sum of two angles calculated above from 180 degrees, enabling UAV to make the turn autonomously.

\subsection{Delivery End}

When the route information request signal has been transmitted to the next node and the node address matches the destination address, UAV has arrived at the target destination. In this case the node transmits a end of delivery signal to UAV and UAV descends to finish the delivery.

\section{Implementation and Validation}

\subsection{Implementation}

Before programming, we performed experiments concerning radio transmission loss values in real world, derive an expression to identify distances between UAV and the nodes. We prepared two sets of a Raspberry Pi 3 and a radio device and considered them to be UAV and smart meters installed in the house. We moved UAV device away from the house, 1 meter at a time, and recorded the radio transmission loss value $L_{B}$, which UAV acquired at

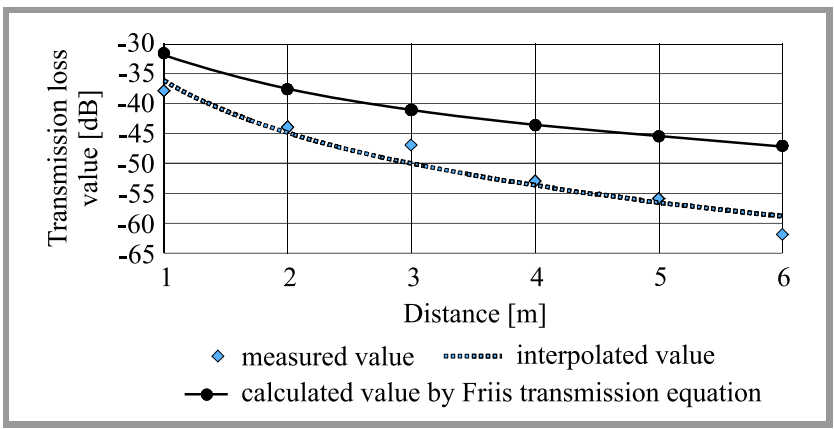

Fig. 12. Radio transmission loss value and distance. the reception of the signal. The calculated radio transmission loss value, the distance in the free space of Eq. (2) presented in Section 4.6 and the value measured in real world are shown in Fig. 12. It was confirmed that the radio transmission loss differed from the theoretical value due to the influence of the atmosphere that acts as the transmission loss medium [8]. This time we measured it in fine weather, but rainfall increases transmission losses even further [9], so it is expected that the loss values will be higher. We assume that UAVs will perform the home delivery service in fine weather, so the effects of rain do not have to be taken into consideration.

If the distance is short, the difference between the calculated value and the measured radio transmission loss values is small. The longer the distance, the larger the difference between the calculated and the measured values. While the results are inaccurate when UAV is far away from the node, it has been confirmed that this distance measuring method may be applied for the purpose of home deliveries performed by UAV.

We derived an approximate curve from the measurement data and defined an expression (7), where e is a bottom of the natural logarithm:

$$
d=\mathrm{e}^{\frac{L_{B}+36.084}{-12.69}} .
$$

We wrote programs to implement the position sensing smart meter radio devices, using Eq. (7) obtained in the above experiment. For the UAV side, we handled sending and receiving signals, programmed positioning and movement control of mobile devices, and enabled the provision of results of navigation control calculations. As far as radio devices of house node side are concerned, we implemented functions enabling them to generate, send and receive those signals.

\subsection{Validation}

We setup four sets comprising a Raspberry Pi 3 and a wireless device each. One set is considered to be UAV, while three remaining sets are used as smart house meters (network nodes). The question was whether UAV and the nodes are capable of transmitting right signals to the specific address. We also validated that the calculation results were correct and that the movement control signals were generated and worked as intended. We run the program and checked the output. In addition, a reverse calculation using spreadsheet software was performed to check whether the output results are correct. The reverse calculation was possible without acquiring the radio transmission loss value, by setting it in each node beforehand.

The result shows that right signals were transmitted and received between the designated nodes (Figs. 13 and 14). The results of positioning and turning calculations were almost the same as the forecast value. Because the discrepancies originate from the difference in the number 


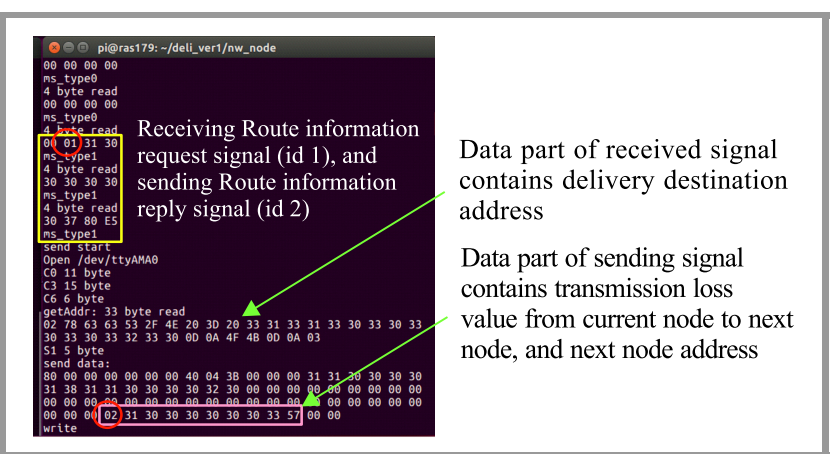

Fig. 13. Validation result 1 .

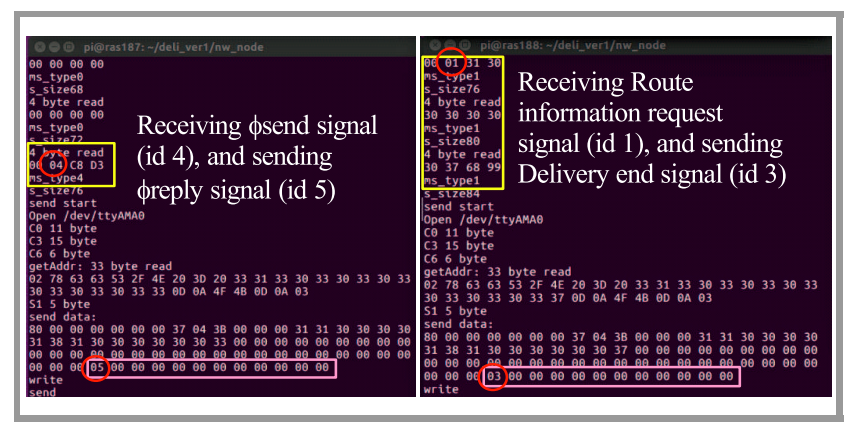

Fig. 14. Validation result 2.

of significant digits, the result indicates that the program we implemented is able to confirm that our proposal has worked well.

\section{Conclusion}

We proposed a routing protocol to establish a safe route for UAV navigation, using a smart meter network. We also proposed methods for position measurement and movement control, enabling UAVs to perform autonomous home deliveries. In the proposed method, distances between UAV and nodes are calculated from the radio transmission loss value. UAV is controlled by determining distance based on the severity of radio transmission loss that occurs while exchanging signals.

Some issues still exist that need to be solved before implementing this system. For example, procedures applied in a scenario in which no electric waves sent by nodes can be detected must be worked out, as must be procedures relied upon to reduce UAV's altitude, track its position or search for another route when no signal is received at all. Safe take-offs and landings are a source of concern as well. We will also establish a method that will change the route if any abnormalities are detected, in order to avoid crashing UAV. All those issues need to be addressed before an autonomous home delivery service relying on UAVs is commissioned.

For future work, we will implement all functions of the proposed method and will experimentally confirm power consumption achieved while using the proposed method and while relying on GPS and map-based navigation.

\section{References}

[1] "IPv6 routing protocol for low power and Lossy networks (RPL)", Routing Over Low power and Lossy networks (ROLL) WG, IETF, RFC 6550, March 2012 [Online]. Available: https://tools.ietf.org/ $\mathrm{html} / \mathrm{rfc} 6550$

[2] “Amazon Prime Air" [Online]. Available: https://www.amazon.com/ Amazon-Prime-Air $/ \mathrm{b}$ ?ie $=$ UTF8\&node $=8037720011$ (accessed Oct. 29, 2018).

[3] "Wing", Google Project [Online]. Available: https://x.company/ projects/wing/ (accessed Oct. 29, 2018).

[4] K. Miyazaki, M. Mochizuki, K. Murao, and N. Nishio, "Crossassistive approach for PDR and Wi-Fi positioning", in Proc. of the 2014 ACM Int. Joint Conf. on Pervas. and Ubiqui. Comput. UbiComp'14, Seattle, WA, USA, 2014, pp. 777-786 (doi: 10.1145/2638728.2641303).

[5] "Ad hoc On-Demand Distance Vector (AODV) Routing", IETF, RFC 3561, July 2003 [Online]. Available: https://tools.ietf.org/ $\mathrm{html} / \mathrm{rfc} 3561$

[6] "Optimized Link State Routing Protocol (OLSR)", IETF, RFC 3626, Oct. 2003 [Online]. Available: https://tools.ietf.org/html/rfc3626

[7] H. Narumi, Y. Shiraishi, and O. Takahashi, "A reliable cluster-based routing algorithm for MANET", in Proc. of the Int. Worksh. on Informat. IWIN 2009, Honolulu, Hawaii, USA, 2009, pp. 44-51, 2009 [Online]. Available: http://www.infsoc.org/conference/ iwin2009/IWIN2009-Proceedings_v2.pdf

[8] Rec. ITU-R P.676-5, "Attenuation by atmospheric gaps”, ITU, 2001 [Online]. Available: https://itu.int/dms_pubrec/itu-r/rec/p/ R-REC-P.676-5-200102-S!!PDF-E.pdf

[9] Rec. ITU-R P.838-2, "Specific attenuation model for rain for use in prediction methods", ITU, 2003 [Online]. Available: https://itu.int/ dms_pubrec/itu-r/rec/p/R-REC-P.838-2-200304-S!!PDF-E.pdf

[10] K. Ueda and T. Miyoshi, "Autonomous navigation control of UAV using wireless smart meter devices", in Proc. of the Inform. and Commun. Technol. Forum ICTF 2018, Graz, Austria, 2018.

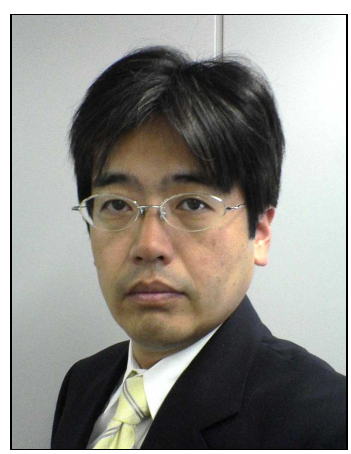

Kiyoshi Ueda received his B.E. and M.E. degrees in Electrical Engineering from Keio University, Yokohama, Japan, in Electrical Engineering from Keio University, Yokohama, Japan, in 1987 and 1989, respectively. He received his Ph.D. degree in Information Science and Electrical Engineering from Kyushu University in 2010. After joining NTT in 1989, he was engaged in research and development work focusing on digital switching software, with a particular emphasis placed on designing distributed switching node management software. He was an active member of ITUT SG11 and SG4, where he contributed to TMN. He was also responsible for research and development of peer-to-peer network service software. Since 2014, he has been a professor at the College of Engineering, Nihon University. He received the Best Paper Award from the IEICE communication society in 2008. He is a member of IEEE, IEICE and IPSJ.

E-mail: ueda.kiyoshi@nihon-u.ac.jp

College of Engineering

Nihon University

Koriyama, Japan 


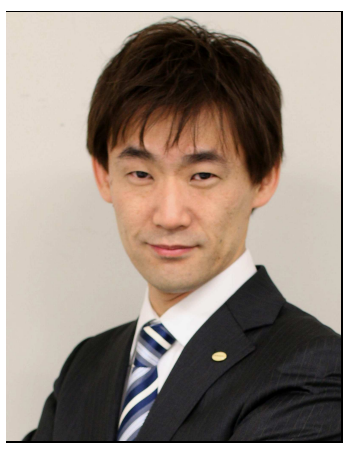

Takumi Miyoshi received his B.E., M.E., and Ph.D. degrees in Electronic Engineering from the University of Tokyo, Tokyo, Japan, in 1994, 1996, and 1999, respectively. He started his career as a research associate at the Global Information and Telecommunication Institute, Waseda University, where he worked from 1999 to 2001. He is currently a Professor at the De- partment of Electronic Information Systems, College of Systems Engineering and Science, Shibaura Institute of Technology, Saitama, Japan. He was a visiting scholar in Laboratoire d'Informatique de Paris 6 (LIP6), Sorbonne Université, Paris, France, from 2010 to 2011. His research interests include content delivery networks, overlay networks, as well as mobile ad hoc and sensor networks.

E-mail: miyoshi@shibaura-it.ac.jp

College of Systems Engineering and Science Shibaura Institute of Technology

Saitama, Japan 